\title{
Molecular Imaging of Inflammation in Aortic Aneurysmal Disease
}

\author{
Alan B. Chan • Eric L. Kaijzel • C. W. G. M. Löwik • \\ Jeroen Essers
}

Published online: 11 November 2011

(C) Springer Science+Business Media, LLC 2011

\begin{abstract}
The high mortality rate of diseases of the aorta has its foundation in imaging methods that define anatomy and disease burden but less so upon the diagnosis of asymptomatic conditions, rate of aneurysm expansion, or prediction of rupture. However, anatomical features can now be co-localized with molecular and physiological activity. The advancement of nanoparticles based upon iron oxide will also serve to bring a trio of magnetic, radionuclide, and optical imaging modalities together. The combinations of these technologies are still at the preclinical refinement stage but already enzyme-activatable probes have been used to interrogate different stages of aneurysmal
\end{abstract}

\section{A. B. Chan}

Percuros B.V.,

Enschede, The Netherlands

\section{A. B. Chan • E. L. Kaijzel • C. W. G. M. Löwik}

Department of Endocrinology and Metabolic Diseases, Leiden University Medical Center,

Leiden, The Netherlands

\section{J. Essers}

Department of Cell Biology and Genetics,

Cancer Genomics Centre (CGC), Erasmus MC,

PO Box 2040, 3000CA, Rotterdam, The Netherlands

J. Essers

Department of Surgical Oncology, Erasmus MC,

PO Box 2040, 3000CA, Rotterdam, The Netherlands

J. Essers

Department of Radiation Oncology, Erasmus MC,

PO Box 2040, 3000CA, Rotterdam, The Netherlands

J. Essers $(\bowtie)$

Erasmus MC,

Room Ee669, Dr. Molewaterplein 50,

3015 GE, Rotterdam, The Netherlands

e-mail: j.essers@erasmusmc.nl disease. Like many disease areas where in vivo optical imaging may play a major role in the future, unravelling and management of aortic aneurysmal disease will progress through better understanding of its pathophysiology. This will translate into new clinical applications led by targetspecific probes and the use of nanoparticle technology.

Keywords Molecular imaging $\cdot$ Inflammation $\cdot$ Aortic aneurysms $\cdot$ Targets $\cdot$ Nanoparticles $\cdot$ Multimodality

\section{Introduction}

Aortic disease is a complex, but potentially deadly disorder, where misdiagnosis is often high. The ability to be able to discern the specific ailment means the difference between surgical intervention and drug therapy. In particular, many physicians have difficulty distinguishing between an aortic aneurysm and an aortic dissection, which are indeed the two most common diseases of the aorta. New molecular imaging techniques may provide a path to earlier detection of symptoms by targeting specific inflammation targets.

Diseased aortic tissue is characterized by chronic inflammation of the aortic wall, degeneration of the smooth muscle cells in the aortic wall, followed by destruction of connective tissue. The diseased tissue is weak and is devoid of sufficient elasticity to stretch and contract optimally. The first indication of this abnormality may be a localized enlargement in the area of weakness and is referred to as an aneurysm. Aortic tissue may also tear, even if the aorta is not enlarged, in a process known as dissection. Tearing of the inner layer of the vessel wall allows blood to leak into the middle layer of the aorta, separating the inner and outer layers.

The causes and risk factors for aortic aneurysms also fall into a number of different categories. Bicuspid aortic 
disease is a manifestation usually attributed as being congenital. Inherited conditions such as Marfan's syndrome and Ehlers-Danlos syndrome [1] demonstrate weakening of the walls of the major arteries, including the aorta. There are also other connective tissue disorders such as Marfanoid, which can lead to the same symptoms. Atherosclerosis changes the lining of the artery wall that may affect oxygen and nutrient flow to the aortic wall tissues. Thus, the result of tissue hardening followed by breakdown may lead to the development of an aneurysm. Miscellaneous infectious and inflammatory conditions such as Takayasu disease [2], though rare and of unknown etiology, can impact upon aortic disease development. Physical trauma, such as an intense blow to the chest or abdomen, can also directly damage the aorta.

This review will focus upon the inflammatory aspects that underpin the pathological processes during development of an aneurysm. The recent developments in the use of molecular imaging will be explored, and especially the diagnostic potential of optical imaging techniques, will be discussed.

\section{Aortic Aneurysms and Inflammation}

Imaging of aneurysms has tended historically to quantify the size of an aneurysm based upon surveillance of anatomical features. However, the pathophysiology of aneurysm formation is associated with an inflammatory cell infiltrate and enzymatic degradation of the vessel wall [3]. Biological markers of inflammation have been associated with risk of aortic rupture [4]. Thus, targeting vascular inflammation through molecular imaging techniques might provide valuable functional information about aneurysms. Recent work has also shown that uptake of magnetic nanoparticles at the luminal surface of patients with highrisk aortic aneurysms correlated with thrombus leukocyte infiltration levels and proteolytic enzyme activity [5]. In the abdominal aorta, the destruction of elastic laminae is characteristic of aneurysm formation. This elastinolysis correlates with overexpression of various elastolytic enzymes, including matrix metalloproteinases (MMPs) 9 and 12 [6-8], and cathepsins S and K [9, 10]. Proteaseactivatable NIRF probes, so-called smart optical probes, are now available (Perkin Elmer; Waltham, MA, USA) for preclinical research applications. Protease-sensing optical probes are autoquench fluorescent probes that convert from a nonfluorescent state to a fluorescent state by proteolytic activation of lysosomal cysteine or serine proteases like cathepsins. By inserting enzyme-specific peptide stalks between the carrier and the fluorochromes the utility of these probes has been extended to other enzymes such as MMPs. The availability of protease-activatable probes may lead to the eventual development of such optical imaging agents as probes for use in the clinic.

\section{Imaging Modalities for Measuring Inflammation in Aortic Aneurysms}

Risk of rupture is an important parameter to consider in terms of management of aortic aneurysms, therefore diagnostic testing is the first step in establishing a treatment strategy. The most promising of these tests involve the use of classical imaging to detect morphological and metabolic changes that would identify patients with an increased risk for disease progression. Imaging modalities such as ultrasonography (US), contrast-enhanced CT angiography (CTA), and MRI are able to provide anatomical information. Aortic aneurysms provide an attractive target for molecular imaging because of their relatively large size as compared with atherosclerotic lesions. The addition of extra information regarding pathophysiology and molecular events would certainly provide an earlier time point prognosis of aneurysm instability, which in turn will lead to better management of the disease. Molecular and functional imaging will lead the charge toward better biomarkers and for more target-specific probes. Moreover, imaging of aortic aneurysms will be improved by combining an anatomical reference together with PET or optical imaging $[11,12 \bullet]$. The use of target-specific contrast agents and/or nanoparticles [13•] will also serve to deliver, initially in animal models, better prognosis of aneurysms.

\section{Imaging Anatomical Features}

Ultrasonography is a cost-effective, highly sensitive and specific imaging technique, which is the method of choice for the detection and follow-up of aortic aneurysms. The technique is used to determine the size of the aneurysm and to predict its expansion. Three-dimensional (3D) ultrasonography has also been employed to improve spatial definition. However, the technique cannot account for the individual variability in the expansion of aneurysms. A promising development has been the use of targeted agents directed against important pathological processes in the aneurysm wall during inflammation. Anti-P-selectin antibodies bound to perfluorocarbon-filled microbubbles have been developed to target P-selectin in activated platelets $[14,15]$. P-selectin plays an essential role in the initial recruitment of leukocytes (white blood cells) to the site of injury during inflammation.

CTA is primarily used for preparatory work before endovascular surgery. A number of anatomical parameters are derived to give informed treatment decisions by 
determining the feasibility of endovascular repair. Beyond this, CTA does have the disadvantage of requiring ionizing radiation and the administration of iodinated contrast agents.

In contrast, MRI uses non-ionizing radiation and can acquire images with high-resolution 3D anatomical features (sub-millimeter), good temporal resolution, and excellent soft tissue contrast. Magnetic resonance angiography (MRA) can be obtained either with fast gradient echo sequences after an injection of gadolinium, or with time-offlight sequences that do not require an injection but have a longer acquisition time. Moreover, MRI can provide an insight into the degree of inflammation by measuring the presence of endogenous iron in macrophages and neutrophils that contain hemosiderin [16•].

\section{Molecular Imaging}

\section{PET/SPECT/CT Imaging}

An imaging modality that offers a better understanding of the pathophysiology of aneurysms is PET. Several reports have highlighted the potential role of fluorine 18-fluorodeoxyglucose (FDG) for the assessment of various aortic diseases based on FDG accumulation. In patients, a strong correlation was found between increased FDG uptake and the progression of aneurysm size, emphasizing that FDG-PET is clinically feasible and available [17]. Furthermore, the stage of plaque development and the level of glucose metabolism within atherosclerotic plaques [18] can be correlated. Accumulation of active macrophages has also been shown to increase the uptake of tracer [19]. However, FDG-PET alone is limited by the low spatial resolution and a lack of morphological information. One way in which this can be overcome is through the use of dual-modality PET/CT, which has emerged as a new diagnostic technique for vascular applications [11].

The implementation of PET/CT has also embraced the use of nanoparticles [20•] on these systems. Their utilization when targeted to macrophages in the form of nanoparticle-based PET reporters has recently been reported. The abundance and pivotal role of macrophages in aneurysm formation $[3,21,22]$ makes them an interesting and clinically relevant imaging target. PET-CT imaging was performed in a mouse model of aortic aneurysm using ${ }^{18} \mathrm{~F}$-cross-linked iron oxide (CLIO) nanoparticles which showed that trace concentrations were detectable [23•]. Thus, PET-CT imaging with ${ }^{18} \mathrm{~F}$-CLIO nanoparticles allows quantitation of macrophage content.

MMPs play a major role in the growth of aortic aneurysms and of their subsequent rupture. Razavian et al. [24•] demonstrated in an animal model that radiotracers with specificity for activated MMPs could be used to detect and quantify MMP activation by microSPECT/CT.

\section{Molecular MRI}

Molecular MRI now offers the ability to image cellular and subcellular level events. Targeted MRI contrast agents have been developed to enable imaging of low levels of expressed targets in vivo. Furthermore, nanoparticles that act as novel contrast agents have been reported that can be potentially developed for diagnostic purposes. Other specialized MRI probes have been developed to image enzyme activity in vivo. These and other advances in molecular MRI offer great promise for the future and have significant potential for clinical translation.

MRI contrast agents based upon ultra small, superparamagnetic iron oxide (USPIO) particles have recently been used [25•] to demonstrate imaging of the inflammatory process involved in aortic aneurysms. USPIO particles are known to accumulate in macrophages in atherosclerotic lesions. A method to assess USPIO uptake within the site of the aneurysm in patients was performed by measurement of semi-quantitative $\mathrm{T} 2$ and $\mathrm{T} 2 *$ relaxation times. Although this study involved only a limited number of patients, it demonstrated the potential of molecular MRI to evaluate biological processes involved with inflammation of the aortic wall and highlights the fact that USPIO contrast agents are clinically available.

It was recently demonstrated in an animal model that inflammation associated with aneurysms could be evaluated noninvasively with MRI and the use of activatable contrast agents. Myeloperoxidase (MPO) is an enzyme found in neutrophils and macrophages and has the potential to be an interesting biomarker for detecting aneurysms. DeLeo et al. [26] evaluated the use of an MPO-sensing activatable MRI contrast agent as a biomarker of the inflammatory neutrophils and macrophages present in a carotid artery brain aneurysm animal model. They were able to visualize aneurysmal inflammation in the brain using clinical strength MRI. A paramagnetic MPO substrate, di-5hydroxytryptamide of gadopentetate dimeglumine, was used to specifically enhance the MRI signal. We are not aware of any similar work relating to molecular imaging of MPO activity in vivo for aortic aneurysms, however, the activation of macrophages or neutrophils where myeloperoxidase is secreted has been reported [27, 28].

A recently developed $[29,30]$ MRI contrast agent, P947, to target MMPs in vivo was evaluated by Bazeli et al. [31]. They showed that MRI using P947 allows detection of MMP activity within the inflammatory wall of experimental abdominal aortic aneurysms. 


\section{Optical Imaging}

Optical molecular imaging is a recently introduced technique that can monitor molecular processes in vivo. From its potential in preclinical studies, it has already shown promise as a tool that will complement classical (nuclear) diagnostic imaging where anatomical references can be superimposed upon molecular events in the format of a multimodality imaging platform. Recently, tumor-specific intraoperative fluorescence imaging has been reported for ovarian cancer in patients [32••].

The preclinical optical probes most extensively tested in cardiovascular animal models have been on MMP activity. MMP activity has been shown in animal models $[12 \bullet, 33 \bullet]$ using an MMP-activatable probe, MMPSense (Perkin Elmer; Waltham, MA, USA). Moreover, Kaijzel et al. [12•] showed that increased MMP activity could already be detected in fibulin-4 (FBLN4) heterozygous mice even before the aneurysm had actually formed (Fig. 1). 3D coregistration of the optical image with $\mathrm{CT}$ pinpointed the MMP activity to the aortic arch (Fig. 2). Fluorescent lifetime of the probe also correlated with and confirmed the location and specificity of MMP activity.

Cathepsin activity has also been documented with respect to cardiovascular disorders [34•]. Chen et al. [35] demonstrated in vivo that cathepsin $\mathrm{B}$, and potentially other proteases, may serve as a biomarker for vulnerable plaques in an animal model. More recently, direct in vivo evidence from studies on mice showed that cathepsin $\mathrm{S}$-induced elastolysis accelerates arterial and aortic valve calcification in chronic renal disease [36]. This non-targeted enzymeactivatable approach has revealed various new findings. However, the identification of new biomarkers of inflammation with target-specificity will be more sought after in the future for early detection of aortic aneurysms. Vascular endothelial growth factor receptor (VEGFR) expression was found to increase in aortic aneurysm mouse models in a diameter-dependent fashion [37•] during in situ NIRF imaging. This may open the door toward clinical VEGFR expression whole body imaging strategies, which could improve real-time monitoring of aneurysmal disease.

\section{Multimodality Imaging Using Nanoparticles}

Polymer-coated iron oxide nanoparticles can be used as imaging contrast agents whereby different imaging moieties can be conjugated for use in a number of modality settings. Two of the nanoparticles, Feraheme and aminoSPARK, are commercially available but have been chemically modified to achieve a specific function. The aminoSPARK nanoparticles are conjugated to near-infrared fluorescent (NIRF) tags giving the structure both optical and MRI properties. PET isotopes can also be conjugated onto the nanoparticles. Furthermore, the use of iron oxide particles also has applications as MRI contrast agents as the core can also have a structure that makes it superparamagnetic, thus making it compatible for MRI studies.

Human ferritin nanoparticles (HFn) conjugated to an Arg-Gly-Asp (RGD) peptide were recently described in mouse carotid and abdominal aortic aneurysm models

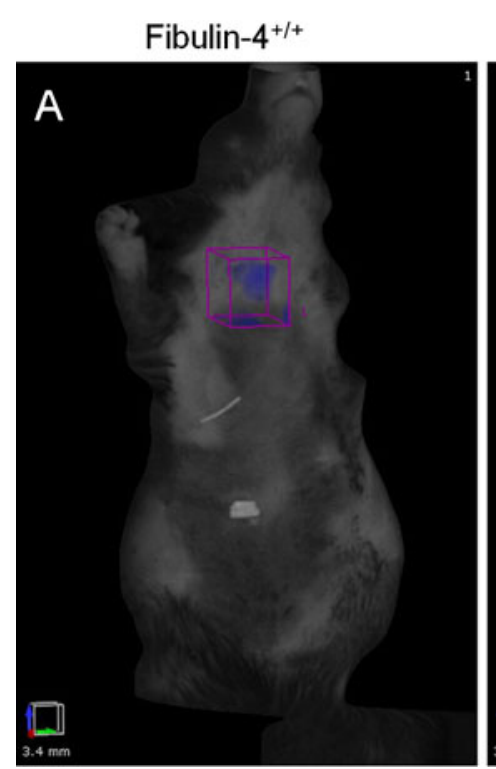

Fig. 1 Three-dimensional registration of MMP upregulation using MMPsense 680 in live fibulin-4 animals using fluorescence molecular tomography imaging. A gradual increase in fluorescence, and thus MMP activation, is already seen in the heterozygous pre-aneurysmal
Fibulin-4+/R

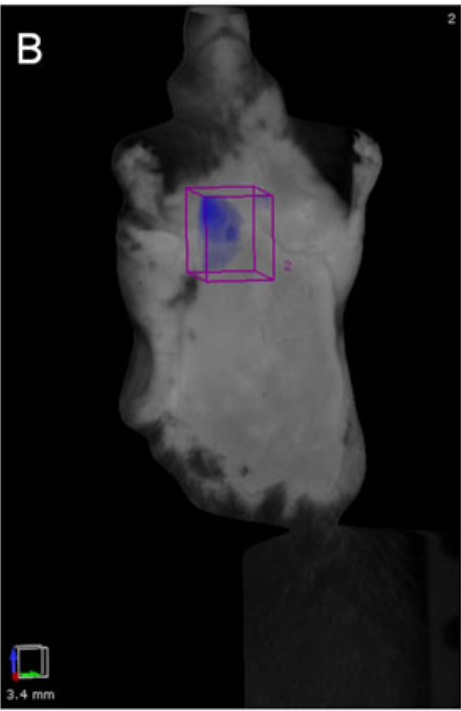

aortic lesions of the fibulin- $4^{+/ R}$ mice (b) compared to wild-type fibulin- $4^{+/+}$animals (a). Homozygous aneurysmal fibulin- $4^{\mathrm{R} / \mathrm{R}}$ mice with a 2-3 fold dilated thoracic aortic arch (c) show a much higher fluorescence level [12•]. (Images provided by R. Ridwan) 


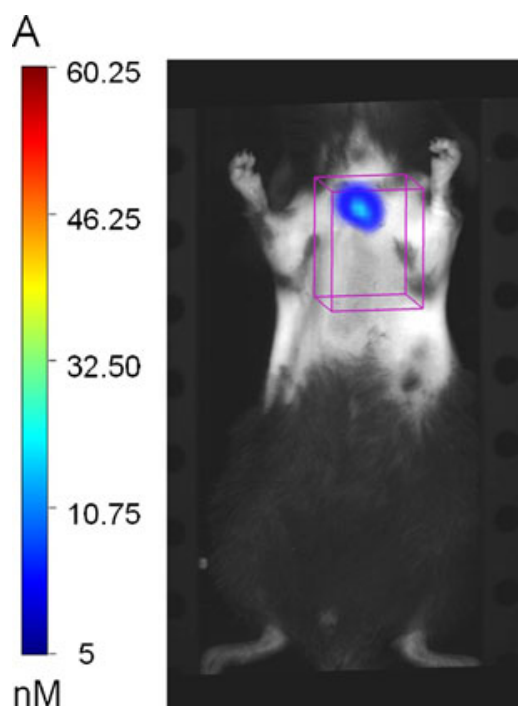

Fig. 2 Complementary anatomical (CT) and optical molecular imaging of pre-aneurysmal lesions in aneurysmal fibulin- 4 mice. a Near-infrared fluorescence images of MMPSense 680 in vivo at 20 hours after injection of a FBLN4 knockdown mouse projected onto the mouse image. b 3D fluorescence-mediated tomography (FMT) and CT co-registration. To reliably identify the region of interest, FMT imaging was hybridized with CT imaging for anatomic reference.

\section{B}

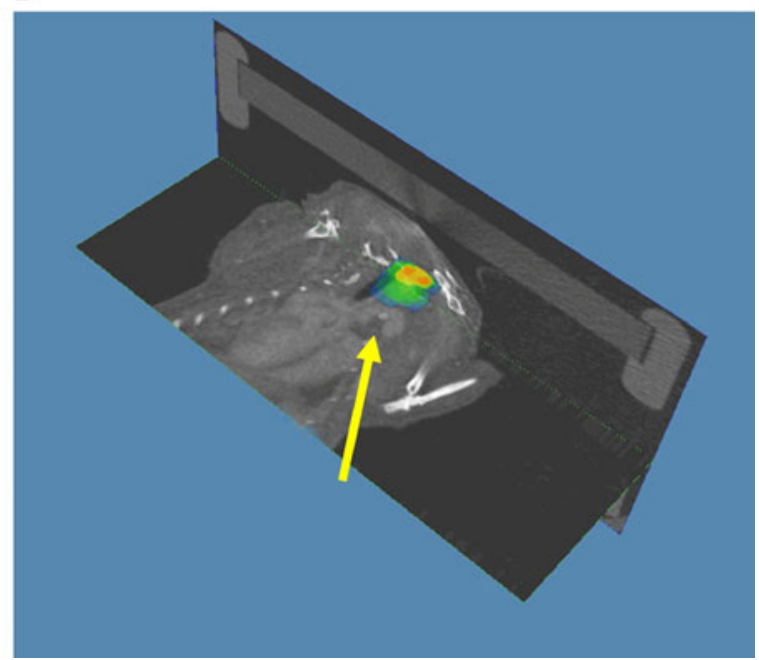

Within both datasets, fiducial points were tagged to define their X-Y-Z coordinates. Using these coordinates, FMT and CT data are fused. While the aorta of the FBLN4 knockdown mouse does not show anatomical signs of aneurysm formation (indicated with the yellow arrow), optical molecular imaging already indicated increased protease activity in the aortic arch compared to normal animals [12•]. (FMT-CT coregistration image provided by J. Dijkstra)
[13•]. The RGD-HFn structure and HFn alone were both chemically modified to accommodate a NIRF label for in vivo imaging. It was found that RGD-HFn showed significantly higher signal than HFn alone in the aortic aneurysm models and also when compared to non-diseased regions. Histology showed that RGD-HFn co-localized with macrophages in aortic aneurysm lesions. The target specificity of the RGD-HFn structure will allow more comprehensive detection of aneurysmal vascular disease.

The use of cross-linked iron oxide nanoparticles for PET-CT imaging with ${ }^{18} \mathrm{~F}$-CLIO demonstrated quantitation of macrophage content in a mouse aortic aneurysm model [23•]. This dual-modality approach enabled a reduction in the nanoparticle dose to clinical levels $(4.5 \mathrm{mg}$ of iron oxide/ $\mathrm{kg}$ body weight, which is lower than the recommended clinical dose for Feraheme). This would have the beneficial effect of being less toxic if the approach translates toward the clinic. The same laboratory also showed that fusion of radionuclide-based molecular (PET) and structural CT modalities could be integrated with fluorescence molecular tomography (FMT). The trio of modalities was integrated and visualized together through a nanoparticle common platform [38•].

\section{Conclusions and Future Perspectives}

Nowadays, aneurysms can be identified with US or CT and can be treated by open or endovascular surgery once they have formed. However, the combination of their small size, unpredictability of rupture, and non-linear expansion means that anatomical imaging alone is insufficient. Indeed, the mortality rate continues to rise and the disease is often under-diagnosed in cases where sudden death occurs. In particular, these new tools, as also addressed by the Food and Drug Administration Critical Path Initiative, are needed to increase the speed, efficiency, and cost-effectiveness of early diagnosis and for drug development. The unmet needs are very apparent when one considers that methylene blue and indocyanine green (ICG) are the only fluorescent-based imaging probes that are FDA-approved. ICG was approved in the late 1950s. The clinical recommendations for the monitoring of molecular events in patients with aortic aneurysms were made in 2005 [39]. It mentions molecular MRI, FDG-PET, and gives a future perspective to the use of nanoparticles. Optical imaging is absent from this list as it is a recently introduced technique and remains still limited for use in preclinical models. However, it is clear that the future clinical interest for treatment and monitoring of aortic aneurysms would be of imaging molecular markers using target-specific approaches in combination with anatomical imaging. The promise of target-specific optical markers was recently given prominence in a clinical study of patients with advancedstage ovarian cancer [32••]. These data were the first to demonstrate the success of using intraoperative tumorspecific fluorescence imaging. This study also has relevance for other diseases as the early detection means 
improved prognosis and more options for therapeutic and intraoperative intervention.

MMP and cathepsin activity have been monitored in animal studies using activatable probes but these are not specific for any particular target. Two recent publications have provided evidence of potential molecular imagingtargeted approaches for aortic aneurysm disease. VEGFR expression was shown to be concordant with aortic aneurysm growth [37•] during NIRF in vivo imaging in mice. A genetically engineered human protein cage conjugated to a RGD peptide demonstrated imaging of vascular inflammation [13•]. The latter also opens the door for nanoparticle and multimodality imaging. Anatomical imaging can provide co-localization of morphology with molecular function of aneurysmal events. The development of radiotracers with novel contrast properties for MRI, CT, or US will enhance the multimodality element to provide for new insights into the pathophysiology of aortic aneurysms. Iron oxide particles will also most likely be at the forefront of a move toward novel nanoparticle technologies. Feraheme is a carbohydrate-coated iron-oxide nanoparticle that was recently approved by the FDA in the US for the treatment of iron deficiency anemia in adult patients with chronic kidney disease. A number of groups are riding on the back of this approval by exploring Feraheme analogous, particles that are optimized to target macrophages to enable eventual fasttrack into clinic $[23 \bullet, 40]$. Nahrendorf et al. [38•] took a $\mathrm{PET} / \mathrm{CT} /$ optical approach incorporating the clinical PET tracer ${ }^{18} \mathrm{~F}$ and a fluorochrome for optical studies. Sadat et al. [25•] used USPIO contrast agents that have proved so effective for MRI in animal models to demonstrate that clinical aortic wall inflammation using USPIO-enhanced MRI is feasible.

The potential clinical impact lies in the fact that several molecular changes can already be monitored in vivo before the aneurysm has actually formed. Implementation of these novel molecular imaging techniques in humans will provide a diagnostic tool to predict aneurysm formation in patients [41•]. An example is the fluorescence molecular tomographic imaging of elevated protease activity in vivo in FBLN4 knockdown mice in which increased MMP activity could already be monitored with near-infrared fluorescence probes before the aneurysm had actually formed [12•]. At present, these FMT systems are only suited for experimental research on relatively small animal models. However, intravascular near-IR fluorescence catheters are promising tools for diagnostic imaging of protease activity in the vascular system $[42,43]$ since these catheters are applicable to humans and do not require large imaging equipment. In addition to conventional imaging modalities, these new diagnostic tools that interrogate the aneurysmatic lesion could potentially serve to test pathophysiological hypothe- ses to identify the risk while the disease remains undetected and to evaluate novel therapeutic strategies. Future work will answer whether these imaging approaches can further improve our ability to diagnose the disease, assess treatment efficacy, and allow clinical translatability.

Disclosure A. B. Chan: consultant to Softscan Healthcare Group; E. L. Kaijzel: none; C.W.G.M. Löwik: none; and J. Essers: none.

\section{References}

Papers of particular interest, published recently, have been highlighted as:

- Of importance

•- Of major importance

1. Callewaert B, Malfait F, Loeys B, et al. Ehlers-Danlos syndromes and Marfan syndrome. Best Pract Res Clin Rheumatol. 2008;22:165-89.

2. Mason JC. Takayasu arteritis - advances in diagnosis and management. Nat Rev Rheumatol. 2010;6:406-15.

3. Shimizu K, Mitchell RN, Libby P. Inflammation and cellular immune responses in abdominal aortic aneurysms. Arterioscler Thromb Vasc Biol. 2006;26:987-94.

4. Lindholt JS, Vammen S, Fasting H, et al. The plasma level of matrix metalloproteinase 9 may predict the natural history of small abdominal aortic aneurysms. A preliminary study. Eur J Vasc Endovasc Surg. 2000;20:281-5.

5. Nchimi A, Defawe O, Brisbois D, et al. MR imaging of iron phagocytosis in intraluminal thrombi of abdominal aortic aneurysms in humans. Radiology. 2010;254:973-81.

6. Thompson RW, Holmes DR, Mertens RA, et al. Production and localization of 92-kilodalton gelatinase in abdominal aortic aneurysms. An elastolytic metalloproteinase expressed by aneurysm-infiltrating macrophages. J Clin Invest. 1995;96:31826.

7. Birkedal-Hansen H. Proteolytic remodeling of extracellular matrix. Curr Opin Cell Biol. 1995;7:728-35.

8. Sakalihasan N, Delvenne P, Nusgens BV, et al. Activated forms of MMP2 and MMP9 in abdominal aortic aneurysms. J Vasc Surg. 1996;24:127-33.

9. Sukhova GK, Shi GP, Simon DI, et al. Expression of the elastolytic cathepsins $\mathrm{S}$ and $\mathrm{K}$ in human atheroma and regulation of their production in smooth muscle cells. J Clin Invest. 1998;102:576-83.

10. Sukhova GK, Shi GP. Do cathepsins play a role in abdominal aortic aneurysm pathogenesis? Ann N Y Acad Sci. 2006;1085:161-9.

11. Kuehl H, Eggebrecht H, Boes T, et al. Detection of inflammation in patients with acute aortic syndrome: comparison of FDG-PET/ CT imaging and serological markers of inflammation. Heart. 2008;94:1472-7.

12. - Kaijzel EL, van Heijningen PM, Wielopolski PA, et al. Multimodality imaging reveals a gradual increase in matrix metalloproteinase activity at aneurysmal lesions in live fibulin-4 mice. Circ Cardiovasc Imaging 2010;3:567-77. A paper that shows in vivo MMP activity through dual-modality noninvasive tomographic imaging and CT. The co-registration of the two modalities presented early detection of increased MMP activity, even before the aneurysm had actually formed.

13. - Kitagawa T, Kosuge H, Uchida M, et al. RGD-Conjugated Human Ferritin Nanoparticles for Imaging Vascular Inflammation 
and Angiogenesis in Experimental Carotid and Aortic Disease. Mol Imaging Biol 2011. A study that demonstrates the use of human ferritin nanoparticles with a RGD target, which significantly enhances vascular inflammation and angiogenesis imaging in experimental models of aortic inflammation.

14. Lindner JR, Song J, Christiansen J, et al. Ultrasound assessment of inflammation and renal tissue injury with microbubbles targeted to P-selectin. Circulation. 2001;104:2107-12.

15. Villanueva FS. Molecular imaging of cardiovascular disease using ultrasound. J Nucl Cardiol. 2008;15:576-86.

16. - Klink A, Hyafil F, Rudd J, et al. Diagnostic and therapeutic strategies for small abdominal aortic aneurysms. Nat Rev Cardiol 2011;8:338-47. A nice overview of diagnostic imaging approaches and therapeutic intervention for tackling small abdominal aortic aneurysms.

17. Sakalihasan N, Van DH, Gomez P, et al. Positron emission tomography (PET) evaluation of abdominal aortic aneurysm (AAA). Eur J Vasc Endovasc Surg. 2002;23:431-6.

18. Rudd JH, Warburton EA, Fryer TD, et al. Imaging atherosclerotic plaque inflammation with [18F]-fluorodeoxyglucose positron emission tomography. Circulation. 2002;105:2708-11.

19. Ogawa M, Ishino S, Mukai T, et al. (18)F-FDG accumulation in atherosclerotic plaques: immunohistochemical and PET imaging study. J Nucl Med. 2004;45:1245-50.

20. • Chacko AM, Hood ED, Zern BJ, et al. Targeted Nanocarriers for Imaging and Therapy of Vascular Inflammation. Curr Opin Colloid Interface Sci 2011;16:215-27. This article documents recent advances in the area of image-guided translation of targeted nanocarrier diagnostics and therapeutics in the treatment of vascular inflammation.

21. Davies MJ. Aortic aneurysm formation: lessons from human studies and experimental models. Circulation. 1998;98:193-5.

22. Tieu BC, Lee C, Sun H, et al. An adventitial IL-6/MCP1 amplification loop accelerates macrophage-mediated vascular inflammation leading to aortic dissection in mice. J Clin Invest. 2009;119:3637-51.

23. - Nahrendorf M, Keliher E, Marinelli B, et al. Detection of macrophages in aortic aneurysms by nanoparticle positron emission tomography-computed tomography. Arterioscler Thromb Vasc Biol 2011;31:750-57. A paper that shows PET-CT imaging using iron oxide nanoparticles that can quantitate macrophage content in a mouse model for aortic aneurysms.

24. - Razavian M, Zhang J, Nie L, et al. Molecular imaging of matrix metalloproteinase activation to predict murine aneurysm expansion in vivo. J Nucl Med 2010;51:1107-15. By using radiotracers specific for activated MMPs, this study shows that MMP-targeted imaging provides unique information on the molecular properties of an aneurysm that are related to expansion.

25. - Sadat U, Taviani V, Patterson AJ, et al. Ultrasmall superparamagnetic iron oxide-enhanced magnetic resonance imaging of abdominal aortic aneurysms - a feasibility study. Eur J Vasc Endovasc Surg 2011;41:167-74. A demonstration in a small cohort of patients that USPIO-enhanced MRI is possible for the detection of aortic wall inflammation.

26. DeLeo III MJ, Gounis MJ, Hong B, et al. Carotid artery brain aneurysm model: in vivo molecular enzyme-specific MR imaging of active inflammation in a pilot study. Radiology. 2009;252:696703.

27. de Vega CM, Esteban M, Quintana JM, et al. Search for serum biomarkers associated with abdominal aortic aneurysm growth - a pilot study. Eur J Vasc Endovasc Surg. 2009;37:297-9.

28. Houard X, Touat Z, Ollivier V, et al. Mediators of neutrophil recruitment in human abdominal aortic aneurysms. Cardiovasc Res. 2009;82:532-41.

29. Lancelot E, Amirbekian V, Brigger I, et al. Evaluation of matrix metalloproteinases in atherosclerosis using a novel noninvasive imaging approach. Arterioscler Thromb Vasc Biol. 2008;28:42532.

30. Amirbekian V, Aguinaldo JG, Amirbekian S, et al. Atherosclerosis and matrix metalloproteinases: experimental molecular MR imaging in vivo. Radiology. 2009;251:429-38.

31. Bazeli R, Coutard M, Duport BD, et al. In vivo evaluation of a new magnetic resonance imaging contrast agent (P947) to target matrix metalloproteinases in expanding experimental abdominal aortic aneurysms. Invest Radiol. 2010;45:662-8.

32. •-van Dam GM, Themelis G, Crane LM, et al. Intraoperative tumor-specific fluorescence imaging in ovarian cancer by folate receptor-alpha targeting: first in-human results. Nat Med 2011. This article represents the first full paper to report on tumorspecific intraoperative fluorescence imaging in humans where folate receptor- $\alpha$ targeting in ovarian cancer was used. Tumorspecific intraoperative fluorescence imaging opens the door toward improved staging and debulking efforts during surgery which will ultimately improve prognosis.

33. - Sheth RA, Maricevich M, Mahmood U. In vivo optical molecular imaging of matrix metalloproteinase activity in abdominal aortic aneurysms correlates with treatment effects on growth rate. Atherosclerosis 2010;212:181-87. An in vivo imaging evaluation of MMP activity that shows a direct, linear relationship between proteolytic activity and aneurysmal growth and that it can be performed endovascularly.

34. - Quillard T, Croce K, Jaffer FA, et al. Molecular imaging of macrophage protease activity in cardiovascular inflammation in vivo. Thromb Haemost 2011;105:828-36. A recent review that describes in vivo imaging of macrophages and protease activity in cardiovascular inflammatory diseases.

35. Chen J, Tung $\mathrm{CH}$, Mahmood U, et al. In vivo imaging of proteolytic activity in atherosclerosis. Circulation. 2002;105:2766-71.

36. Aikawa E, Aikawa M, Libby P, et al. Arterial and aortic valve calcification abolished by elastolytic cathepsin S deficiency in chronic renal disease. Circulation. 2009;119:1785-94.

37. • Tedesco MM, Terashima M, Blankenberg FG, et al. Analysis of in situ and ex vivo vascular endothelial growth factor receptor expression during experimental aortic aneurysm progression. Arterioscler Thromb Vasc Biol 2009;29:1452-57. A study that shows VEGFR expression imaging in experimental aortic aneurysm models, which supports similar earlier findings that this marker could be used as a surrogate for monitoring disease progression.

38. • Nahrendorf M, Keliher E, Marinelli B, et al. Hybrid PET-optical imaging using targeted probes. Proc Natl Acad Sci U S A 2010;107:7910-15. A publication that highlights the possibilities of multimodality imaging (PET/CT/optical) using nanoparticle technology as a common platform.

39. Hirsch AT, Haskal ZJ, Hertzer NR, et al. ACC/AHA 2005 Practice Guidelines for the management of patients with peripheral arterial disease (lower extremity, renal, mesenteric, and abdominal aortic): a collaborative report from the American Association for Vascular Surgery/Society for Vascular Surgery, Society for Cardiovascular Angiography and Interventions, Society for Vascular Medicine and Biology, Society of Interventional Radiology, and the ACC/AHA Task Force on Practice Guidelines (Writing Committee to Develop Guidelines for the Management of Patients With Peripheral Arterial Disease): endorsed by the American Association of Cardiovascular and Pulmonary Rehabilitation; National Heart, Lung, and Blood Institute; Society for Vascular Nursing; TransAtlantic InterSociety Consensus; and Vascular Disease Foundation. Circulation. 2006;113:e463-654.

40. Weissleder R, Kelly K, Sun EY, et al. Cell-specific targeting of nanoparticles by multivalent attachment of small molecules. Nat Biotechnol. 2005;23:1418-23. 
41. Buxton DB, Antman M, Danthi N, et al. Report of the national heart, lung, and blood institute working group on the translation of cardiovascular molecular imaging. Circulation 2011;123:2157-63. A recent summary by a working group that reviewed state-of-the-art technologies, their challenges, clinical needs, and specific recommendations for cardiovascular imaging.
42. Jaffer FA, Calfon MA, Rosenthal A, et al. Two-dimensional intravascular near-infrared fluorescence molecular imaging of inflammation in atherosclerosis and stent-induced vascular injury. J Am Coll Cardiol. 2011;57:2516-26.

43. Jaffer FA, Vinegoni C, John MC, et al. Real-time catheter molecular sensing of inflammation in proteolytically active atherosclerosis. Circulation. 2008;118:1802-9. 